\title{
Effects of aspiration pneumonia on the intensive care requirements and in-hospital mortality of hospitalised patients with acute cerebrovascular disease
}

Adil Can Güngen ${ }^{1}$, Yusuf Aydemir ${ }^{1}$, Belma Dogan Güngen², Esra Ertan Yazar ${ }^{3}$, Orhan Yağız ${ }^{4}$, Yeşim Güzey Aras $^{2}$, Hatice Gümüşs, Ünal Erkorkmaz ${ }^{6}$

\author{
${ }^{1}$ Department of Pulmonology, Sakarya University, Sakarya, Turkey \\ 2Department of Pulmonology, Sakarya University, Training and Research Hospital, \\ Sakarya, Turkey \\ ${ }^{3}$ Department of Pulmonology, Yedikule Chest Diseases and Chest Surgery Training \\ Hospital, Istanbul, Turkey \\ ${ }^{4}$ Department of Neurology, Istanbul Research and Training Hospital, Istanbul, Turkey \\ ${ }^{5}$ Department of Neurology, Türkan Özilhan State Hospital, Izmir, Turkey \\ ${ }^{6}$ Department of Biostatistics, Medical Faculty, Sakarya University, Sakarya, Turkey
}

Submitted: 28 July 2015

Accepted: 1 November 2015

Arch Med Sci 2017; 13, 5: 1062-1068

DOI: 10.5114/aoms.2016.61011

Copyright $\odot 2016$ Termedia \& Banach

\section{Abstract}

Introduction: In this study, we aimed to evaluate the effects of the development of aspiration pneumonia (AP) on the intensive care unit (ICU) requirements and in-hospital mortality of patients hospitalised in the neurology ward due to an acute cerebrovascular accident (CVA).

Material and methods: Five hundred and three patients hospitalised in the neurology ward following an acute CVA were retrospectively analysed. The patients were divided into two groups: those with AP (group 1) and those without AP (group 2). Demographic characteristics and physical and radiological findings, including the localisation, lateralisation and aetiology of the infarction, in addition to ICU requirements and mortality, were evaluated.

Results: Aspiration pneumonia was detected in 80 (15.9\%) patients during the in-hospital stay. Transfer to the ICU for any reason was required in $37.5 \%$ of the patients in group 1 and $4.7 \%$ of those in group $2(p<0.001)$. In-hospital mortality occurred in $7.5 \%$ and $1.4 \%$ of the patients in group 1 and group 2 , respectively $(p=0.006)$. The incidence of AP was highest in patients with an infarction of the medial cerebral artery (MCA) $(p<0.001)$. The AP was associated with older age $(p<0.001)$, hypertension $(p=0.007)$, echocardiography findings $(p=0.032)$ and the modified Rankin Scale (mRS) score $(p<0.001)$. Conclusions: Our findings suggest that the requirement rate for transfer to the ICU and the mortality rate appear to be significantly higher in patients with a diagnosis of AP. Precautions should be taken, starting from the first day of hospitalisation, to decrease the incidence of AP in patients with acute CVA, focusing especially on older patients and those with a severe mRS score.

Key words: aspiration pneumonia, cerebrovascular accident, intensive care unit, mortality.

\section{Introduction}

Stroke is the second leading cause of death and is a major cause of disability worldwide [1]. Approximately one-fourth of patients with

\section{Corresponding author:}

Assist. Prof. Yusuf Aydemir Department of Pulmonology Sakarya University Training and Research Hospital 54100 Sakarya, Turkey Phone: +90 5056369494 E-mail: dryaydemir@yahoo. com 
a stroke die within 1 month after the stroke, and almost one-third and $50 \%$ die within 6 months and 1 year, respectively [2]. Stroke-related pneumonia is a major cause of increased mortality, with the rate of early mortality in patients who developed pneumonia reported as between $10 \%$ and $37 \%$ [3]. The possible risk factors predisposing patients to aspiration and respiratory infection following a stroke are the placement of a nasogastric tube, immobilisation, localisation of the lesion and the presence of dysphagia [3-5]. However, there are no data in the available literature on how aspiration pneumonia (AP) affects intensive care unit (ICU) requirements and mortality.

In this study, we aimed to evaluate the effects of the development of AP on ICU requirements and in-hospital mortality among patients hospitalised in the neurology ward due to an acute cerebrovascular accident (CVA). We believe that this study can significantly contribute to the literature, as it evaluates the effects of the development of pneumonia on the consequences of the disease in a large series of patients hospitalised following an acute CVA.

\section{Material and methods}

Between January 2012 and April 2013, 503 consecutive patients who were hospitalised in the Istanbul Research and Training Hospital neurology clinic with a diagnosis of ischemic stroke were included in the study. Their demographic characteristics, physical examination findings and radiological examination results, including the localisation, lateralisation and aetiology of the infarction, were retrospectively evaluated. All the patients underwent a routine chest X-ray, echocardiography and blood tests. Thoracic computed tomography (CT) was performed in the presence of newly developed infiltrates on the chest radiograph and the presence of respiratory system findings, such as a cough, fever, tachypnoea and pathological respiratory sounds. The radiological assessment of the thoracic CT was performed by two different experienced pulmonologists. In cases of no diagnostic agreement between the reviewers, the patient was excluded from the study. The criteria (coexistence must be present) for the diagnosis of AP were: 1) the presence of appropriate clinical and radiological findings, including thoracic CT findings, and 2) a nurse or relative of the patient witnessing the aspiration. Patients with accompanying immunosuppression, malignancy or known structural lung disease were not included in the study. The patients were divided into two groups: those with AP (group 1) and those without AP (group 2).

The indication for admission to the ICU was based on the Glasgow Coma scales, with a score of 7 resulting in transfer to the ICU, as standard. The evaluation for ICU transfers were assessed independently from AP. After the decision of ICU transfer, the presence or absence of AP was noted.

The subtypes of ischemic stroke were classified according to the original Trial of Org 10172 Acute Stroke Treatment (TOAST) criteria [6]. The five major categories of the TOAST classification are: large-artery atherosclerosis, including large-artery thrombosis and artery-to-artery embolisms; cardio-embolisms; small artery occlusions; stroke of determined causes; and stroke of undetermined causes. For a diagnosis of stroke with an undetermined origin, one of the following two items must be present:

1) No aetiology, despite an extensive examination.

2) Most likely cause undetermined, with $>1$ possible cause detected.

The definitions of the subtypes were made according to the risk factor profiles, clinical features and results of the diagnostic tests of the patients. CT, magnetic resonance imaging, vascular imaging (carotid duplex, transcranial Doppler), an electrocardiogram and echocardiography were performed, and pro-thrombotic syndromes were assessed.

The echocardiography findings were classified into four groups, with echocardiogram results carrying a risk of stroke accepted as: 1) thrombus in the left atrium/atrial appendix, 2) left ventricular thrombus, 3) dilated cardiomyopathy and 4) akinetic/hypokinetic left ventricular segment.

The modified Rankin scale (mRS) was used to grade the functional ambulation/disability of the patients during their hospitalisation, as follows [7]: 0 - No symptoms.

1 - No marked disability, with the patient able to perform daily activities and tasks.

2 - Mild disability, with the patient unable to perform ordinary tasks and activities but capable of undertaking daily tasks.

3 - Moderate disability, with partial help needed to perform tasks but capable of walking unaided.

4 - Severe disability, with the patient unable to walk without help and unable to attend own bodily needs without help.

5 - Very severe disability, with the patient bed-ridden, incontinent and requiring continuous care and attention.

6 - Death.

Lesion areas were categorised according to the areas of the vessels: medial cerebral artery (MCA), anterior cerebral artery, posterior cerebral artery and basilar artery and cerebellar arteria (superior and inferior). The MCA infarcts were further classified according to the dimensions of the lesion (MCA1: lacunar infarcts; MCA2: between lacunar 
infarcts-total MCA infarct; and MCA3: total MCA infarcts).

The mortality data included mortality that occurred during the hospital stay and during the 1-month post-discharge period. Local research ethics committee approval was obtained for this study.

\section{Statistical analysis}

The statistical analysis was performed using the commercial software IBM SPSS Statistics, version 23.0. (IBM Corp., Armonk, NY). As the distributions of the variables were not normal according to the Kolmogorov-Smirnov normality test, a Mann-Whitney U-test was used to compare the age and mRSs of the two groups. The categorical variables were compared using Pearson's, Yates' or Fisher's exact $\chi^{2}$ test. The categorical variables are presented as counts and percentages. Spearman's correlation coefficient was used to analyse the relation between AP and the other variables. A multivariate logistic regression model was implemented to determine the ICU transfer, mRS and other covariates associated with AP. A $p$-value of $<0.05$ was considered statistically significant.

\section{Results}

A total of 503 patients who were hospitalised in the neurology ward with a diagnosis of

Table I. General characteristics of the study population

\begin{tabular}{|c|c|}
\hline Parameter & Value \\
\hline Total patients & 545 \\
\hline Excluded patients due to: & 42 \\
\hline $\begin{array}{l}\text { The lack of exact diagnosis of } \\
\text { pneumonia }\end{array}$ & 17 \\
\hline The lack of thorax CT & 12 \\
\hline The lack of reviewers agree & 5 \\
\hline $\begin{array}{l}\text { Accompanying structural lung } \\
\text { disease (COPD, bronchiectasis, } \\
\text { asthma and malignancy) }\end{array}$ & 25 \\
\hline Included patients & 503 \\
\hline Age & $68.18 \pm 13$ \\
\hline Sex (male) & $265(53 \%)$ \\
\hline Smoking & 201 (40\%) \\
\hline Hypertension & $328(65.2 \%)$ \\
\hline Diabetes & $163(32.4 \%)$ \\
\hline ICU transfer & $50(9.9 \%)$ \\
\hline Mortality & $12(2.4 \%)$ \\
\hline
\end{tabular}

ischemic stroke were analysed. The mean age of the patients was $68.18 \pm 13$ years. Of these, 265 $(52.7 \%)$ were male, and 238 (47.3\%) were female. The demographic characteristics of the patients are shown in Table I.

Aspiration pneumonia was detected in 80 (15.9\%) patients during their in-hospital stay. The mortality rate was $7.5 \%$ in group 1 and $1.4 \%$ in group 2 ( $p=0.006$; Table II).

While 30 of $80(37.5 \%)$ patients with AP were enrolled in the ICU, 20 of 423 (4.7) patients without AP were enrolled in the ICU. The average time for onset of AP was 3.4 days (median: 3.0, min: 1, $\max : 8)$.

The WBC, platelet and neutrophil counts, CRP and ESR values were significantly higher in the patients in whom pneumonia was detected than in those in whom it was not $(p<0.0001$, for all). The patients' clinical and laboratory characteristics are presented in Table II.

A univariate analysis of the demographic and clinical outcomes showed a relationship between AP and age, hypertension, echocardiographic findings, mRS and TOAST criteria. Advanced age was a significant risk factor for pneumonia $(p<0.001$, $r=0.181$ ). The AP was more frequent in patients with hypertension ( $p=0.007, r=0.121)$ and with high-risk echocardiography findings ( $p=0.021$, $r=0.103$ ). However, AP was not associated with sex, cigarette smoking, diabetes or atrial fibrillation (Table III).

Aspiration pneumonia was seen most frequently in patients with an mRS score of 5 (58.75\%) and, in descending order of frequency, in patients with a score of 4 (20\%), 3 (17.5\%) and 1 (3.75\%) $(p<0.001, r=0.391)$. The median mRS score was 5 and 3 in the AP group and non-AP group, respectively. A positive correlation was observed between aspiration pneumonia and $\mathrm{mRS}(p<0.001$; $r=0.391$ ) (Tables II and III).

When we consider the relationship between the cranial magnetic resonance findings and pneumonia, our correlation studies revealed a significant correlation between MCA1 ( $p=0.001$, $r=-0.145)$, MCA3 $(p<0.001, r=0.210)$ and AP. We found no correlation between the other areas of the vessels and AP (Table III).

When analysed according to TOAST criteria, pneumonia was observed more frequently in patients with large-artery atherosclerosis (21.7\%) and cardio-embolisms (21\%) $(p=0.005)$. After hospital admission, transfer to the ICU for any reason was required in $37.5 \%$ of patients in group 1 and $4.7 \%$ of patients in group $2(p<0.001)$. A positive correlation was found between ICU transfer and AP ( $p<0.001, r=0.401)$. The multivariate analysis showed that advanced age and a severe mRS score were associated with AP (Table IV). 
Table II. Comparison of patients with and without aspiration pneumonia

\begin{tabular}{|c|c|c|c|}
\hline \multirow[t]{2}{*}{ Parameter } & \multicolumn{2}{|c|}{ Aspiration pneumonia } & \multirow[t]{2}{*}{$P$-value } \\
\hline & $\begin{array}{l}\text { Present group } 1 \\
\quad(n=80)\end{array}$ & $\begin{array}{l}\text { Absent group } 2 \\
(n=423)\end{array}$ & \\
\hline Age & $73.31 \pm 11.16$ & $67.21 \pm 13.10$ & $<0.001$ \\
\hline Sex (male) & $43(53.8)$ & $222(52.5)$ & 0.835 \\
\hline Smoking & $31(38.8)$ & $170(40.2)$ & 0.810 \\
\hline WBC $\left[\times 10^{9} / 1\right]$ & $14.61 \pm 4.53$ & $6.79 \pm 2.24$ & $<0.001$ \\
\hline Neutrophil count $\left[\times 10^{9}\right]$ & $11.41 \pm 4.39$ & $4.20 \pm 1.91$ & $<0.001$ \\
\hline Red blood cells & $4.20 \pm 0.4$ & $4.28 \pm 0.4$ & 0.088 \\
\hline Platelets & $310.5 \pm 91.2$ & $272.4 \pm 67.1$ & $<0.001$ \\
\hline $\mathrm{CRP}[\mathrm{mg} / \mathrm{l}]$ & $20.25 \pm 21.3$ & $0.58 \pm 0.5$ & $<0.001$ \\
\hline $\mathrm{ESR}[\mathrm{mm} / \mathrm{h}]$ & $81.39 \pm 28.6$ & $27.39 \pm 20.3$ & $<0.001$ \\
\hline Hypertension & $62(77.5)$ & $261(61.7)$ & 0.007 \\
\hline Diabetes & $30(37.5)$ & $133(31.4)$ & 0.288 \\
\hline Echocardiographic findings & $24(30)$ & $79(18.7)$ & 0.032 \\
\hline Atrial fibrillation & $16(20)$ & $60(14.2)$ & 0.245 \\
\hline \multicolumn{4}{|l|}{ Carotid Doppler findings: } \\
\hline Normal & $43(53.8)$ & $264(62.4)$ & \multirow[t]{3}{*}{0.012} \\
\hline Symptomatic & $36(45)$ & $129(30.5)$ & \\
\hline Asymptomatic & $1(1.2)$ & $30(7.1)$ & \\
\hline \multicolumn{4}{|l|}{ mRS: } \\
\hline 0 & 0 & $2(0.5)$ & \multirow[t]{7}{*}{$<0.001$} \\
\hline 1 & $3(3.8)$ & $46(10.9)$ & \\
\hline 2 & 0 & $94(22.2)$ & \\
\hline 3 & $14(17.5)$ & $153(36.2)$ & \\
\hline 4 & $16(20)$ & $75(17.7)$ & \\
\hline 5 & $47(58.8)$ & $52(12.3)$ & \\
\hline 6 & 0 & $1(0.2)$ & \\
\hline $\mathrm{mRS}$ & $4.30 \pm 1.0$ & $2.98 \pm 1.2$ & $<0.001$ \\
\hline$m R S>2$ & $77(96.3)$ & $281(66.4)$ & $<0.001$ \\
\hline \multicolumn{4}{|c|}{ Cranial magnetic resonance findings: } \\
\hline MCA1 & $1(1.3)$ & $60(14.1)$ & \multirow[t]{7}{*}{$<0.001$} \\
\hline MCA2 & $36(45)$ & $164(38.8)$ & \\
\hline MCA3 & $19(23.8)$ & $29(6.9)$ & \\
\hline ACA & $3(3.8)$ & $27(6.4)$ & \\
\hline PCA & $6(7.5)$ & $35(8.3)$ & \\
\hline BA & $9(11.3)$ & $74(17.5)$ & \\
\hline SA & $6(7.5)$ & $34(8.0)$ & \\
\hline \multicolumn{4}{|l|}{ TOAST criteria: } \\
\hline Large-artery atherosclerosis & $43(53.8)$ & $155(36.6)$ & \multirow[t]{5}{*}{0.005} \\
\hline Cardio-embolism & $16(20)$ & $60(14.2)$ & \\
\hline Small artery occlusion & $8(10)$ & $72(17.0)$ & \\
\hline Other determined cause & $12(15)$ & $113(26.7)$ & \\
\hline Undetermined cause & $1(1.3)$ & $23(5.4)$ & \\
\hline ICU transfer & $30(37.5)$ & $20(4.7)$ & $<0.001$ \\
\hline Mortality & $6(7.5)$ & $6(1.4)$ & 0.006 \\
\hline
\end{tabular}

Data were shown as mean $\pm S D$. Deviation and $n$ (\%), WBC - white blood cells, CRP - C-reactive protein (normal $<0.9)$, ESR - erythrocyte sedimentation rate (normal <20), MCA - medial cerebral artery, ACA - anterior cerebral artery, PCA - posterior cerebral artery, BA-basilar artery, SA - arteria cerebellaris, ICU - intensive care unit, $m R S$ - modified Rankin scale. 
Table III. Correlations between aspiration pneumonia and other variables

\begin{tabular}{|c|c|c|}
\hline \multirow[t]{2}{*}{ Parameter } & \multicolumn{2}{|c|}{ Aspiration pneumonia } \\
\hline & $r$ & $P$-value \\
\hline Age & 0.181 & $<0.001$ \\
\hline Sex (male) & 0.009 & 0.835 \\
\hline Smoking & -0.011 & 0.810 \\
\hline Hypertension & 0.121 & 0.007 \\
\hline Diabetes & 0.047 & 0.289 \\
\hline Echocardiographic findings & 0.103 & 0.021 \\
\hline Atrial fibrillation & 0.059 & 0.184 \\
\hline Carotid doppler findings & 0.043 & 0.334 \\
\hline $\mathrm{mRS}$ & 0.391 & $<0.001$ \\
\hline $\mathrm{mRS}>2$ & 0.241 & $<0.001$ \\
\hline \multicolumn{3}{|c|}{ Cranial magnetic resonance findings: } \\
\hline MCA1 & -0.145 & 0.001 \\
\hline MCA2 & 0.047 & 0.297 \\
\hline MCA3 & 0.210 & $<0.001$ \\
\hline ACA & -0.041 & 0.363 \\
\hline PCA & -0.010 & 0.817 \\
\hline BA & -0.062 & 0.168 \\
\hline SA & -0.007 & 0.871 \\
\hline TOAST criteria & -0.160 & $<0.001$ \\
\hline ICU transfer & 0.401 & $<0.001$ \\
\hline Mortality & 0.146 & $<0.001$ \\
\hline
\end{tabular}

The comparison of the demographic and clinical characteristics of the two groups and the disease consequences are shown in Tables II and III.

\section{Discussion}

Previous studies demonstrated that several factors independently increase the risk of death or a poor outcome following an acute stroke [8, 9]. Approximately two-thirds of early death and poor outcomes in acute stroke are attributed to nonmodifiable predictors [3]. The main modifiable factors are early complications, such as pneumo- nia. Stroke units should focus on preventing pneumonia to improve the prognosis of patients with acute strokes [3]. Despite the role of pneumonia in increasing mortality, to the best of our knowledge there are no data on the ICU transfer requirements of patients admitted with a stroke.

Our study was performed to investigate the independent and attributable impact of AP on acute stroke mortality and ICU transfer rates. For the first time, we estimated the association between AP and ICU transfer requirements. We found that $37.5 \%$ of patients with AP and $4.7 \%$ of patients without AP were transferred to the ICU. In our study, the ICU transfer rate and mortality rate increased 8-fold and 5-fold, respectively, in patients who developed AP soon after a stroke.

The incidence of AP among the majority of neurological ICU studies ranged from $9.5 \%$ to $56.6 \%$, whereas it ranged from 3.9 to $12 \%$ in stroke unit studies $[3,8]$. In our study, the rate of AP during the in-hospital stay was $15.9 \%$.

The rate of early mortality due to pneumonia, one of the major complications following CVAs, has been reported to be between $7 \%$ and $37 \%$ $[3,8,10,11]$. Furthermore, CVA-related mortality has been reported to vary depending on the region, race and socioeconomic status. The low rates in developed countries can likely be attributed to good nutrition, pre-defined risk factors with scheduled follow-up and subsequent modification of treatments $[12,13]$. Some studies have demonstrated that the presence of dementia and living alone are associated with mortality after a stroke [12]. In our patient population, the mortality rate due to AP was $7.5 \%$.

Patients who develop pneumonia after a stroke have substantial morbidity and mortality $[3,8$, 11]. Identifying patients with the greatest risk of developing pneumonia after a stroke might help with treatment and prevention. In our study, there was a significant correlation between AP and advanced age, hypertension, high-risk echocardiographic findings, the Rankin score and TOAST criteria in the univariate analysis. On the other hand, we found no significant difference in sex,

Table IV. Multivariate logistic regression model for aspiration pneumonia

\begin{tabular}{|lcccc|}
\hline Parameter & $\beta$ & S.E. of $\beta$ & $P$-value & OR (95\% Cl for OR) \\
\hline Age & 0.042 & 0.013 & 0.001 & $1.043(1.017-1.069)$ \\
\hline Hypertension & 0.460 & 0.331 & 0.164 & $1.585(0.828-3.032)$ \\
\hline Diabetes & 0.510 & 0.304 & 0.093 & $1.666(0.918-3.024)$ \\
\hline Echocardiographic findings & 0.387 & 0.327 & 0.237 & $1.473(0.776-2.796)$ \\
\hline mRS $>2$ & 2.311 & 0.623 & $<0.001$ & $10.089(2.976-34.200)$ \\
\hline ICU transfer & 2.305 & 0.357 & $<0.001$ & $10.022(4.983-20.156)$ \\
\hline
\end{tabular}

ICU - intensive care unit, mRS - modified Rankin scale. 
atrial fibrillation, diabetes, smoking and the carotid Doppler findings between the patients who had versus those who did not have AP. The multivariate analysis showed that elderly patients and those with a high mRS score more frequently developed pneumonia.

The prevalence of abnormal echocardiographic findings in our hospitalised patients was $20.4 \%$, which is comparable to the prevalence reported in a previous study [14]. Aspiration pneumonia was more frequent in patients with high-risk echocardiography findings (30\%) compared to those with normal echocardiography findings (8.7\%, $p=0.032$; Table II).

Pneumonia has been reported to be an important cause of morbidity and mortality, particularly in the elderly population. The causes of the high frequency of pneumonia in the elderly population include impaired elasticity of the lungs with ageing and altered physiological parameters, such as compliance, respiratory muscle strength, mucociliary transport and cough reflexes, as well as altered oropharyngeal colonisation, all of which weaken the immune system [3, 15]. In addition, dysphagia is seen in $\sim 50 \%$ of patients with stroke [16]. Oropharyngeal aspiration due to dysphagia, resulting in pneumonia, has been reported in patients with CVAs. Oropharyngeal colonisation may vary with ageing. Changes in oropharyngeal colonisation can adversely affect both the frequency and course of pneumonia [15]. Several studies have reported an increased risk of aspiration in patients with CVAs, with the placement of a nasogastric tube, malnutrition, female gender, cognitive functional impairment, dysphagia and impaired mobilisation reported to increase respiratory infections [17-20].

The stroke severity measured by the National Institutes of Health Stroke Scale (NIHSS) or the $\mathrm{mRS}$ is a major independent risk factor for stroke-associated pneumonia (SAP). Traditionally, SAP was thought to be secondary to aspiration. However, various clinical studies have shown that aspiration and its related risk factors, such as an impaired level of consciousness and dysphagia, are important risk factors for SAP. Many stroke patients have impaired swallowing mechanisms, which, theoretically, may be related to abnormal dopamine transmission, leading to aspiration of oral content during sleep [3]. Thus, the high incidence of AP in patients with a high score on the $m R S$ seems reasonable.

In another study of the association of AP with the localisation of the lesion, lesions adjacent to the insular cortex and internal capsule increased the development rate of AP [5]. In our study, the most frequently seen localisation of the lesions in patients with AP was the MCA infarction. In the present study, we found a significant positive correlation between MCA3 and AP and a negative correlation with MCA1.

The localisation and dimensions of lesions in cerebrovascular diseases, advanced age and accompanying chronic diseases are unpreventable and eventually result in worsening of clinical conditions. However, AP, which worsens the clinical presentation and increases disability, is preventable, at least in some patients. In such patients, preventive measures, in addition to attentive care and awareness raising among patients' relatives, can help to combat AP. The control of swallowing, implementation of a dysphagia screening protocol, prevention of aspiration, enteric feeding methods, such as a percutaneous gastrostomy tube, administration of prophylactic antibiotics and early mobilisation are recommended to decrease the risk of SAP [3].

Our study has some limitations that should be acknowledged. First, we evaluated the patient's condition only after hospitalisation. Unfortunately, there are no long-term follow-up data. Second, we did not perform clinical and/or instrumental assessments of swallowing, such as a videofluoroscopic test. Third, the NIHSS data were incomplete.

In conclusion, the rate of requirement for transfer to the ICU and the mortality of patients with acute CVAs who developed AP were significantly higher than those of the patients without AP. Procedures should be put in place to identify patients hospitalised with a diagnosis of an acute CVA to decrease the incidence of AP, starting on the first day of hospitalisation.

\section{Conflicts of interest}

The authors declare no conflict of interest.

\section{References}

1. Donnan GA, Fisher M, Macleod M, Davis SM. Stroke. Lancet 2008; 371: 1612-23.

2. Hankey GJ, Jamrozik K, Broadhurst RJ, et al. Five-year survival after first-ever stroke and related prognostic factors in the Perth Community Stroke Study. Stroke 2000; 31: 2080-6

3. Hannawi Y, Hannawi B, Rao CP, et al. Stroke-associated pneumonia: major advances and obstacles. Cerebrovasc Dis 2013; 35: 430-43.

4. Marciniak C, Korutz AW, Lin E, et al. Examination of selected clinical factors and medication use as risk factors for pneumonia during stroke rehabilitation: a case-control study. Am J Phys Med Rehabil 2009; 88: 30-8.

5. Galovic M, Leisi N, Müller M, et al. Lesion location predicts transient and extended risk of aspiration after supratentorial ischemic stroke. Stroke 2013; 44: 2760-7.

6. Kolominsky-Rabas PL, Weber M, Gefeller O, et al. Epidemiology of ischemic stroke subtypes according to TOAST criteria: incidence, recurrence, and long-term survival in ischemic stroke subtypes: a population-based study. Stroke 2001; 32: 2735-40. 
7. Sulter G, Steen C, De Keyser J. Use of the Barthel index and modified Rankin scale in acute stroke trials. Stroke 1999; 30: 1538-41.

8. Koennecke HC, Belz W, Berfelde D, et al. Factors influencing in-hospital mortality and morbidity in patients treated on a stroke unit. Neurology 2011; 77: 965-72.

9. Siebert J, Gutknecht P, Molisz A, et al. Hemodynamic findings in patients with brain stroke. Arch Med Sci 2012; 8: 371-4.

10. Langhorne P, Stott DJ, Robertson L, et al. Medical complications after stroke: a multicenter study. Stroke 2000; 31: 1223-9.

11. Katzan IL, Cebul RD, Husak SH, et al. The effect of pneumonia on mortality among patients hospitalized for acute stroke. Neurology 2003; 60: 620-5.

12. Appelros P, Nydevik I, Viitanen M. Poor outcome after first-ever stroke: predictors for death, dependency, and recurrent stroke within the first year. Stroke 2003; 34: 122-6.

13. Feigin VL, Forouzanfar MH, Krishnamurthi R, et al. Global and regional burden of stroke during 1990-2010: findings from the Global Burden of Disease Study 2010. Lancet 2014; 383: 245-54.

14. Amin $\mathrm{H}$, Aronow WS, Lleva $\mathrm{P}$, et al. Prevalence of transthoracic echocardiographic abnormalities in patients with ischemic stroke, intracerebral hemorrhage, and subarachnoid hemorrhage. Arch Med Sci 2010; 6: 40-2.

15. Stupka JE, Mortensen EM, Anzueto A, Restrepo MI. Community-acquired pneumonia in elderly patients. Aging Health 2009; 5: 763-74.

16. Martino R, Foley N, Bhogal $S$, et al. Dysphagia after stroke: incidence, diagnosis, and pulmonary complications. Stroke 2005; 36: 2756-63.

17. Brogan E, Langdon C, Brookes K, et al. Dysphagia and factors associated with respiratory infections in the first week post stroke. Neuroepidemiology 2014; 43: 140-4.

18. Matsumura T, Mitani Y, Oki Y, et al. Risk factors for the onset of aspiration pneumonia among stroke patients in the recovery stage. Nihon Ronen Igakkai Zasshi 2014; 51: 364-8.

19. Kojima A, Imoto Y, Osawa Y, Fujieda S. Predictor of rehabilitation outcome for dysphagia. Auris Nasus Larynx 2014; 41: 294-8.

20. Brogan E, Langdon C, Brookes K, et al. Respiratory infections in acute stroke: nasogastric tubes and immobility are stronger predictors than dysphagia. Dysphagia 2014; 29: 340-5. 